\title{
Mental Images and Postpartum Depression: Case Study
}

\author{
Dorina Bedhia \\ Clinical Psychologyst at University hospital \\ for obstetrics and Gynecology “Koco Gliozheni” Tirana, Albania
}

\section{Abstract}

Imagination and images refer jointly ability to imagine. Imaginative therapies operate all within an almost real context. In therapeutical experience, the individual goes through almost real experiences before going through the events in reality, acts before acting in reality and this provokes changes in somatic level. The almost real dimension, namely the imaginative dimension, influences the individual, or rather the individual, starting from the imagination changes himself, his beliefs and perceptions. Imagination as therapeutic intervention is sometimes more efficient and more valuable than other therapies. It is also effective in treating a range of psychological symptoms such as insomnia, depression, obesity, cronich pain, various phobias, anxiety and panic , somatic problems. Given the fact that the images are effective in treating a range of psychological symptoms, including depression we want to see if imaginative techniques help improve symptoms of postpartum depression. This case was treated at University Hospital for Obstetric and Gynecology "Koco Gliozheni" Tirane (Albania). A 35 years young mother showed depressive symptoms associated with post-partum condition, as determined by semi-structured interviews and relevant test EDPS, also by psychiatric consultations. Besides the daily psychological support I proposed some imaginative techniques like self-watching, flooding, guided imagery. Imaginative activity in general, in the case in question, was a valid instrument of the difficulties in everyday life. The patient learned to visualize problematic elements of each situation and this resulted an efficient approach. Imagination helped identify schematic components that have contributed to the formation of inappropriate thoughts and exaggerated ideas. It helped in recognition of the patient's emotional reality and modifying this emotional reality. The patient uses images to manage situations different daily life even by telephone follow up. This case study shows that imagery techniques, elaborated through images, facilitate recovery and provide us with a functional interpretation of the event and its consequences. Working with images intended to make the patient able to withstand and manage the pain that bring different situations and to integrate it in the history of personal life. 
Keywords: imaginative techniques, post-partum depression.

\section{Introduction}

\section{Background}

The imagination, images and imaginative techniques have always raised great interest. Imagination and images refer jointly the ability to imagine. Imagination is action (Widman 2004). It is the ability to shape mental images, to transform them, to develop them and to deform them. In Psychological terminology it is a form of thinking that does not follow fixed rules, nor logical relationships but is presented as a reproduction and elaboration of current or past sensory data associated with a certain affective stage. The imagination allows the combination of memories, perceptions, aspirations and real context by creating in mind something new, transformed and re-elaborated thanks to the fantasy. Images are the basis of psychic life. The approach that is used by some orientations and imaginative psychotherapies lies on the fact that some imaginative experiences take on the colors of reality and it is precisely this almost real dimension that acts in changing the individual. The ability to imagine allows the experimentation in this almost real dimension. The almost real dimension, namely the imaginative dimension, succeeds in influencing the individual, or to be more precise, the individual starting from the imagination changes himself, his own beliefs and perceptions. The process that is followed by most of the therapies is from the fantasy to reality.

The imagination as an intervention technique has yielded results in various fields. Most clinicians use imaginative techniques to promote relaxation, to reduce stress, to regain control of unwanted behaviors and to improve health.

They are effective in treating a variety of psychological symptoms such as depression, different phobias, anxiety and panic, sleeplessness, somatic problems.

Imaginative techniques are incorporated into psychoanalytic, psychodynamic and cognitive behavioral theories as supplementary therapeutic methods.

\section{Case Study}

Our patient is transferred to the obstetric-gynecological university hospital 'Koco Gliozheni' Tirana (Albania) from Berat maternity. It is 30-31 week pregnancy and the patient is diagnosed with preeclamsia. She is 35 years old, married and in her first pregnancy. She gives birth to a girl with a weight of 1100 grams, who starts a treatment at the intensive therapy unit at the "Koco Gliozheni" maternity hospital.

The first psychological counseling is requested by the gynecologist and then by the pediatrician who is curing the patient's daughter. The latter asks for the consultation because of the lack of interest shown by the mother to the child. 
The patient complains of persistent headaches, chest pain and breathing difficulties. After examinations with the specialized doctors, the room physician requests a psychological counseling. The first consultation is done about one month after the baby's birth. The patient complains of headaches, breathing difficulties and persistent anxiety that does not allow her to sleep but is trying to suffocate her. She goes very rarely to see the girl because she does not feel good, according the declaration of the patient. She is always accompanied by her mother-in-law and husband who are present at almost all meetings with the patient and they refuse to leave her alone through consultations.

In the upcoming hours and days the situation appears alternately, there are days when she is quiet and other days when she only cries because she does not feel physically fit.

\section{Methodology}

The patient started to follow the consultation regularly every day. During the consultations, there were constantly present either the husband or the mother-in-law of the patient who with all the insistence of the psychologist refused to leave, moreover by taking the approval of the patient. This aspect was counterproductive because it did not allow us to obtain additional information on the patient's family problems. In the presence of the family, the patient complained continuously of physical problems but not for psychological problems. The psychologist noted sleep disorders, fatigue and lack of energy, anxiety, guilt feelings, thoughts of death and suicide.

With the cooperation of the maternity staff we tried to find time when the patient could be consulted alone in order to explore her thoughts and emotions.

Initially I conducted semi-structured interviews that consisted of cognitive questions. These questions are proposed by the National Institute for Health and Clinical Excellence (United Kingdom) (Nice 2007), and are important in screening postpartum.

- Did you feel very sad and hopeless during the last month?

- Did you have little interest or little pleasure in daily activities during the last month?

If the above questions result positive then the third question is raised

- Taking in consideration this situation, do you feel the need for help?

After the answers to the questions were all positive we applied the Edinburgh test for postnatal depression.

The Edinburgh Postnatal Depression Scale (EDPS) is a valuable and effective way of identifying patients at risk for perinatal depression. The EDPS is easy to administer and has proven to be an effective screening tool. Mothers who score above 13 are 
likely to be suffering from a depressive disease of varying severity. The scale indicates how the mother has felt during the previous week.

From the interviews we made and from the respective tests we identified depressive symptoms.

We sought a psychiatric consultant, who based on the assessments made, confirmed the diagnosis of postpartum depression and proceeded with the respective pharmacological therapy.

In addition to pharmacological therapy, the patient continued to have constant psychological support.

But since the communication with the patient was difficult and we reached a point without way out, we thought to use the imagination, starting from the fact that the patient's imagination was quite alive (at least from the description of the scenes of death and suicide which she imagined and outlined).

Self-Watching Technique: This technique uses self-observation to change compulsive behaviors. It is taught to the patient to identify the factors that support and associate compulsive behavior, also are taught the techniques that modify this situation. The patient should keep a behavioral diary in which he should note any compulsive behavior and the circumstances in which this behavior occurs. This helps in identifying events, ideas and feelings that influence the behavior. The patient is assisted in creating the strategies to face and not avoid them. Important in this technique is the identification of alternative satisfaction based on the fact that it is easier to resist to compulsive behaviors when they are anticipated from alternative positive behaviors.

Flooding Tech: Polin (1959) has named it as a shocking therapy. It consists in the imagination of an anxiety situation that is experienced with the maximum intensity including all the emotional dyeing that it causes. This image pushes the patient to the limit of patience and aims to avoid strategies that the patient usually uses in similar situations such as avoiding or leaving.

The directive images were used to build a future-oriented identity. To re-imagine or re-live changes the patient's point of view regarding the reality of the pain.

\section{Discussions And Conclusions}

The period of pregnancy and birth are important events that contribute not only to the birth of a human being such as a child but also to the birth of a new identity of the next mother, particularly from the psychological point of view. Pregnancy is configured as a fundamental process in shaping of the female identity. Like all phases of identity crisis, this stage also has its own conflicts because the mother's personality is tested and faces constant changes and adjustments. The woman can withstand with difficulty this process, and the birth of the baby may worsen the situation, causing humorous disorders from the easiest to the worst. 
The post-partum depression phenomenon in Albania is highly underestimated. There are no good statistics for this phenomenon. Medical staff are not trained to identify these cases and refer them to relevant institutions. The psychological service in maternity is regularly implemented since 5 years.

Patients are still not happy about the psychological support. There is still the mentality that asking for psychological help means being crazy. And these prejudices become even more distinct among the people living in the city and those living in the village.

On top of these limitations, adds the fact that a child's birth is a joyful event and should not bring any kind of problem, and therefore new mothers have difficulty in expressing and showing their problems.

Even when maternity personnel faced such problems, is the mother herself or her family members who try to minimize the problem, not pay attention, even to refuse psychological support or even when they accept it they are not at all cooperative.

Such problems are also encountered in our case. The husband of the patient and her family wanted to be in consultation, and this limited the confession of the woman.

Since verbal communication was blocked at a point without a way out, I decided to use the imagination and the imaginative techniques to understand the problems, motivations and behaviors of the patient, starting from the fact that the imagination helps to face daily difficulties and improve the lifestyle

Images are always associated with emotion. It is a bidirectional relationship. If images produce emotions the latter together with feelings and affections produce imaginative activity, which is used to recognize the individual's cognitive-emotional reality and to modify it if necessary.

Imaginative exposure is used as an alternative to the real-life difficulties. These images are capable of provoking a positive or negative emotional state, transforming some aspects of the external and internal reality, facilitating the identification and analysis of emotional states, cognitive processes, to identify and modify the distorted and irrational beliefs of patients through a change in the degree of anxiety stimulus risk or through a change in the way of assessing the ability to confront.

The directive images used with our patient helped us discover new areas of exploration, such as a depressive episode that occurred six years ago and treated with medicines. Or the conflicting relationship with her husband and her family, or fear of having a child who would have a life full of suffering like her, so she preferred to die together with the baby.

The images helped us to modify the irrational beliefs that led to the appearance of inappropriate emotions. 
The directive images helped us to start the creation of a future-projected identity, a future that includes her child.

The patient began to approach and stay more with her child, and one month later, after daily psychological consultations and psychiatric support, came out of the hospital in a better state.

In a telephone follow-up with the patient, the patient referred us to be better, changed some things in her life. She had been divorced from her husband and had gone to live with her daughter at her parents, she had started a new job. She was happy with the new life she had created, and she continued to consult periodically with mental health institute.

This clinical case is a positive example, which indicates that work with imagery and imaginative techniques is more effective where words are lacking, and where barriers and protective mechanisms are very obvious.

\section{References}

[1] Antonietti,A ., Giorgetti,M. (1993). Pensare attraverso immagini. Vita e Pensieri. Milano

[2] Arbuthnott,Katherine D., Arbuthnott, Deniss W. (2001).Guided imagery andmemory:Implications for psychotherapists. Journal of Counseling Psychology,Vol 48(2),pp.123-132

[3] Beck, C. T. (1996b). Postpartum depressed mothers' experiences interacting with their children. Nursing Research, 45, 98-104.

[4] Boyd, R. C., Pearson, J. L., and Blehar, M. C. (2002). Prevention and treatmentof depression in pregnancy and the postpartum period: summary of a maternal depression roundtable: UA perspective. Arch.Women Ment. Health 4, 79-82.

[5] Campos, A.,Perez ,Fabello,M,J., Gonzalez,MA. (1999).Capacity for mental imagery and its spontaneous use. Perceptual and motor skills. 88(3 Pt 1),pp.856858.

[6] Edwards,D. (1990). Cognitive therapy and the restructuring of early memories through guided imagery .Journal of Cognitive Psychotherapy,4.pp.3350

[7] Giusti, E. (2007). Tecniche imaginative:il teatro interiore nelle relazioni di aiuto. Sovera Multimedia:Roma

[8] Hall,Eric.,Hall,Carol. et al.(2006). Guided imagery:Creative interventions in counseling \& psychotherapy .Sage publications,137pp.

[9] Holmes,Emily.,Mathews,Andrew .(2005). Mental imagery and emotion:A special relationship?. Emotion,Vol 5(4),pp.489-497 
[10] McMahon ,E.Carol., Sheikh,Anees. (2002). Imagination in disease and healing processes.A historical perspective.pp.1-22.In Sheikh,Anees .(2002). Handbook of therapeutic imagery techniques. Amityville,NY,US,Baywood Publishing Co.vii,414 $\mathrm{pp}$

[11] Edited by Vivtoria, Hendrick. (2006) .Psychiatric Disorders in Pregnancy and the Postpartum: Principles and Treatment.

[12] Sheikh,Anees. (2002). Handbook of therapeutic imagery techniques. Amityville,NY,US,Baywood Publishing Co.vii,414 pp.

[13] Utay, Joe.,Miller,Megan. (2006). Guided imagery as an effective therapeutic technique:A brief review of its history and efficacy research. Journal of Instructional Psychology. Vol 33(1),pp.40-43. 